\title{
Efecto de una intervención de enfermería para la disminución del consumo de alcohol de riesgo en adultos mayores
}

\section{Effect of a nursing intervention to reduce risky alcohol consumption in older adults}

\section{Efeito de uma intervenção de enfermagem para reduzir o consumo de álcool de risco em adultos mais velhos}

\author{
Alicia Álvarez-Aguirre ${ }^{1 *}$ \\ https://orcid.org/0000-0001-5538-7634 \\ Leticia Casique-Casique ${ }^{2}$ \\ (iD) https://orcid.org/0000-0002-0532-4819 \\ Benjamín López-Nolasco 3 \\ (iD) https://orcid.org/0000-0003-4566-214X \\ María del Rosario Tolentino-Ferrel ${ }^{4}$ \\ (iD) https://orcid.org/0000-0002-4690-5564
}

1. Universidad de Guanajuato, Campus Celaya Salvatierra. División de Ciencias de la Salud e Ingenierías. Departamento de Enfermería Clínica. Guanajuato, México.

2. Universidad de Guanajuato, Campus Celaya Salvatierra. División de Ciencias de la Salud e Ingenierías. Departamento de Enfermería y Obstetricia. Guanajuato, México

3. Universidad Autónoma del Estado de Hidalgo. Escuela Superior de Tlahuelilpan. Hidalgo México

4. Universidad de Guanajuato, Campus Celaya Salvatierra. División de Ciencias de la Salud e Ingenierías. Departamento de Enfermería y Obstetricia. Guanajuato, México

*Autor para correspondencia: alicia.alvarez@ugto.mx

\section{Resumen}

Introducción: el consumo de alcohol de riesgo en adultos mayores constituye un problema social y de salud, debido a que intensifica el efecto nocivo en ellos, por los cambios biopsicosociales y espirituales como resultado del proceso natural de envejecimiento; en la familia repercute emocional y económicamente en sus integrantes, en las instituciones de salud demanda servicios, genera incremento de consumo de recursos económicos y humanos. Objetivo: evaluar el efecto de una intervención para disminuir el consumo de alcohol de riesgo en adultos mayores. Metodología: estudio cuasi experimental realizado en un Centro Gerontológico en México la muestra fue de 50 participantes por muestreo no probabilístico. La intervención se fundamentó en la Terapia Racional Emotiva Conductual. Se aplicaron el AUDIT y un cuaderno de recogida de datos personales. Se obtuvieron frecuencias, porcentajes, medidas de tendencia central y dispersión, además de la prueba 
Wilcoxon. Resultados: predominó el sexo femenino (58\%), 72\% de los participantes contaban con primaria incompleta, las edades se encontraron entre 60 y 71 años $(\bar{X}=64.42 ; D E= \pm 2.80)$. Al comparar las mediciones del consumo de alcohol se observó significancia estadística $(Z=-6.160, p<.001)$ y la mediana fue menor en la medición post intervención (Mediana pre $=12$ vs Mediana post $=7$ ). Conclusiones: la intervención mostró efecto en la disminución del consumo de alcohol de riesgo.

Palabras clave: Anciano; Alcohol; Intervención; Enfermería (DeCS).

\begin{abstract}
Introduction: alcohol consumption in older adults constitutes a social and health problem, because it intensifies the harmful effect on them, due to biopsychosocial and spiritual changes as a result of the natural aging process; It affects the family emotionally and economically on its members, in health institutions it demands services, generates an increase in the consumption of economic and human resources. Objective: to evaluate the effect of an intervention to reduce risky alcohol consumption in older adults. Methodology: quasi-experimental study carried out in a Gerontological Center in Mexico, the sample was 50 participants by non-probabilistic sampling. The intervention was based on Rational Emotive Behavioral Therapy. The AUDIT and a data card were applied. Frequencies, percentages, measures of central tendency and dispersion were obtained, in addition to the Wilcoxon test. Results: the female sex predominated (58\%), $72 \%$ of the participants had incomplete primary school, the ages were between 60 and 71 years $(\bar{X}=64.42 ; S D= \pm 2.80)$. When comparing the measurements of alcohol consumption, statistical significance was observed $(Z=-6.160, p<.001)$ and the median was lower in the post-intervention measurement (Median pre $=12$ vs Median post $=7$ ). Conclusions: the intervention showed an effect in reducing risky alcohol consumption.
\end{abstract}

Key words: Aged; Alcohol; Intervention; Nursing (DeCS).

Abstrato

Introdução: o consumo de álcool em idosos constitui um problema social e de saúde, pois intensifica os efeitos deletérios sobre eles, devido às mudanças biopsicossociais e espirituais decorrentes do processo natural de envelhecimento; Afeta emocionalmente e economicamente a família em seus membros, nas instituições de saúde demanda serviços, gera aumento no consumo de recursos econômicos e humanos. Objetivo: avaliar o efeito de uma intervenção para reduzir o consumo de risco de álcool em idosos. Metodologia: estudo quase experimental realizado em um Centro Gerontológico do México, a amostra foi de 50 participantes por amostragem não probabilística. A intervenção foi baseada na Rational Emotive Behavioral Therapy. O AUDIT e um cartão de dados foram aplicados. Foram obtidas frequências, porcentagens, medidas de tendência central e dispersão, além do teste de Wilcoxon. Resultados: predominou o sexo feminino (58\%), $72 \%$ dos participantes possuíam o ensino fundamental incompleto, as idades variaram entre 60 e 71 anos ( $\bar{X}=64,42 ; \mathrm{DP}= \pm 2,80$ ). Ao comparar as medidas de consumo de álcool, foi observada significância estatística $(Z=-6,160, p<0,001)$ e a mediana foi menor na medida pós-intervenção (Mediana pre $=12$ vs Mediana post $=7$ ). Conclusões: a intervenção mostrou efeito na redução do consumo de álcool de risco.

Palavras-chave: Idoso; Álcool; Intervenção; Enfermagem (DeCS).

Recibido: 25/03/2020

Aceptado: 15/06/2021 


\section{Introducción}

El consumo de alcohol es una práctica en todo el mundo, con efectos nocivos en la salud y sociedad. Es un hábito que ha evolucionado históricamente desde un acto ilegal, hasta su concepción actual de práctica socialmente aceptada en numerosas culturas ${ }^{(1)}$. Esta conducta como factor de riesgo para la discapacidad, en el mundo ocupa los terceros lugares y los primeros en el continente americano ${ }^{(2,3)}$; persiste a pesar de las consecuencias perjudiciales que tiene sobre el organismo, en un contexto considerado de consumo de riesgo, que representa graves problemas de salud pública y social ${ }^{(4,5)}$ debido a que los problemas físicos y mentales relacionados con el consumo de alcohol de riesgo no se presentan de inmediato sino después de varios años, lo que aumenta la demanda de servicios al sistema de salud y la carga de enfermedad ${ }^{(6)}$. Aunado con el crecimiento de la población de Adultos Mayores (AM), el número de bebedores en riesgo aumenta porque los adultos jóvenes que en este momento tienen consumo de alcohol de riesgo pasarán a la etapa de AM con este hábito ${ }^{(7)}$.

El consumo excesivo de alcohol tiene repercusiones negativas en el contexto en el que vive la persona, afecta su salud física y mental igualmente la de su familia y de las personas que la rodean ${ }^{(8,9)}$. Las situaciones antes descritas se exacerban al tratarse de la población de AM, por los cambios fisiológicos propios del envejecimiento que hacen que experimenten mayor concentración de alcohol en la sangre y deterioro, tales como el envejecimiento de los vasos sanguíneos, el cerebro disminuye su volumen, el riñón muestra disminución moderada de la velocidad de filtración glomerular, la masa muscular disminuye y aumenta su infiltración grasa; lo que incrementa el riesgo de morbilidad en comparación con los adultos más jóvenes (10, 11,12).

En la revisión de literatura se señala que $41 \%$ de AM consumieron alcohol frecuentemente y $20 \%$ ingirieron alcohol con medicamentos ${ }^{(11,13)}$. También se reporta que los adultos de 60 años, tenían consumo de alcohol de riesgo ${ }^{(7,14)}$ y $21.58 \%$ de los adultos mayores de 70 años fueron consumidores de alcohol ${ }^{(6)}$. Se registra que el grupo de entre 35 y 65 años de edad, 30.6\% presentó consumo excesivo de alcohol en el último año, 2\% fueron consumidores con dependencia, $7.2 \%$ consumidores frecuentes y $2.3 \%$ consume diariamente alcohol ${ }^{(15)}$. Por sexo se indica que los hombres mayores de 60 años, presentaron mayor consumo de alcohol que las mujeres $(p<.01)^{(16)}$. Por otra parte, 
reportan que los AM que consumían alcohol $13.8 \%$ necesitaron de un cuidador, la prevalencia de las enfermedades infecciosas respiratorias fue mayor cuando los AM refirieron abuso o dependencia del alcohol en los últimos 12 meses. Además, el consumo de alcohol alguna vez en la vida se asoció con la polifarmacia $(\mathrm{OR}=1.26, \mathrm{IC} 95 \%=1.01-1.57)^{(17)}$.

El consumo de alcohol, por parte de los AM es un problema poco abordado en México ya que las encuestas nacionales de adicciones suelen incluir solo a personas hasta 65 años.

Los factores que favorecen la reducción del consumo de alcohol en esta población son: una salud más pobre, menor ingreso, uso de múltiples medicamentos y disminución de ocasiones de beber; en tanto los factores que mantienen la conducta de riesgo se encuentran: el ser varón, tener educación superior, ingreso económico solvente, contar con redes sociales que fomenten el consumo de alcohol y usar el alcohol para manejar el dolor, duelo, soledad y aislamiento social, entre otros $(11,14,18)$.

Referente a las estrategias para disminuir el consumo de alcohol en AM, la literatura muestra que la duración de las intervenciones es entre uno y tres meses, con seguimiento entre dos y seis meses, la frecuencia de la sesiones es entre una y dos sesiones semanales. En cuanto al enfoque son de tipo educativo, psicológico o psicoeducativo, su contenido incluye retroalimentación de consumo, informan sobre conductas saludables para reducir el consumo de alcohol de riesgo, monitoreo de las metas de consumo de alcohol a través de mensajes de texto, llamadas telefónicas o bien en cada sesión ${ }^{(10,19,20,21)}$.

Concerniente a los resultados de las intervenciones, la revisión de evidencia muestra que al término de la intervención los resultados indicaron una diferencia significativa en el grupo de intervención vs control, en bebedores en consumo de riesgo (66\% vs $88 \%$ ) y consumo excesivo de alcohol (45\% vs 68\%) ${ }^{(19)}$. Otro estudio refirió que $41.7 \%$ de los participantes del grupo control presentaron consumo de alcohol de riesgo en cambio en el grupo experimental fue de $28.6 \%(\mathrm{OR}=0.56, \mathrm{IC} 95 \%=0.16-1.95, p=.36)^{(20)}$. Asimismo, se observaron cambios en la cantidad de bebidas alcohólicas (14.9 vs $12.1, p<.05)$ y consumo de riesgo $(2.8 \text { vs } 2.1, p<.01)^{(10)}$. Los resultados mostraron asociación significativa en la disminución de visitas al médico (-1.14 visitas; $p<.05)$, al departamento de urgencias (16\% vs 25\%; $p<.01)$ y del cuidador (12\% vs $17 \% ; p<.01)^{(21)}$. 
El impacto del consumo de alcohol de riesgo en AM se focaliza en su cuidado, incluyendo la necesidad de un cuidador por la falta de capacidad de realizar las actividades básicas e instrumentales de la vida diaria, entre otros ${ }^{(22)}$. Resulta evidente promover intervenciones para esta población en específico, a fin de disminuir el consumo de alcohol de riesgo y daños a la salud, a través de capacitación de $\mathrm{AM}$ en el consumo responsable ${ }^{(23)}$. Aunado a que esta población es más propensa que los adultos más jóvenes a buscar atención del profesional de salud, lo que abre la puerta para el reconocimiento del consumo de alcohol de riesgo en los $\mathrm{AM}^{(21,24)}$.

La participación del profesional de enfermería es fundamental en la prevención del consumo de alcohol de riesgo, porqué en su quehacer profesional: valora, diagnóstica, orienta, favorece el desarrollo de habilidades para la prevención de conductas de riesgo, así como el control de enfermedades y prevención de sus consecuencias a fin de favorecer una conducta saludable en la persona, familia y comunidad. Por lo que el profesional de enfermería tiene una oportunidad única de detectar y prevenir las repercusiones del consumo de alcohol de riesgo en las esferas biopsicosocial y espiritual, que a menudo son difíciles de intervenir en el AM. El objetivo fue evaluar el efecto de una intervención de enfermería para la disminución del consumo de alcohol de riesgo en adultos mayores.

\section{Metodología}

Estudio de tipo cuantitativo, se abordó un diseño cuasi experimental con un grupo y una medición pre y posintervención. El universo estuvo conformado por el total de AM $(N=145)$ registrados en un Centro Gerontológico de la Región Centro de México. Se realizó un análisis de poder estadístico aplicando la metodología y definiciones sobre el tamaño de efecto de Cohen ${ }^{(25)}$, para una prueba de diferencias de medias, con nivel de significancia de .05 , un poder de $90 \%$ y un tamaño de efecto .80 , considerando una deserción estimada del $10 \%$ obteniendo 50 participantes. Los criterios de elegibilidad fueron: participantes de 60 años y más, con consumo de alcohol de riesgo, que se encontraran registrados en el Centro Gerontológico y que otorgaron su consentimiento informado.

La colecta de los datos se realizó a partir de un cuaderno de recogida de datos personales que incluyó la edad, sexo y escolaridad. Además de la prueba de Identificación de los Trastornos debidos al Consumo de Alcohol [AUDIT]. La prueba AUDIT se encuentra dividida en tres dominios: consumo de alcohol de riesgo que incluye los 
primeros tres ítems; síntomas de dependencia que incorpora del ítem 4 al 6 y consumo perjudicial de alcohol que integra del ítem 7 al 10. La interpretación del puntaje total es de 0-5 puntos sin riesgo; de 6 a 8 puntos consumo de riesgo y de 9 a 40 puntos indica dependencia. Los autores del instrumento reportaron una confiabilidad al Alpha de Cronbach de 0.90 (26), el instrumento fue validado para población mexicana (27).

El proyecto, fue aprobado por el Comité de Investigación de la División de Ciencias de la Salud e Ingenierías del Campus Celaya Salvatierra de la Universidad de Guanajuato con número de registro: CIDSC-3062405 y se contó con la autorización del Centro Gerontológico y consentimiento informado de los participantes. Posterior a ello, en los 145 AM, de forma individual, se aplicó el cuaderno de recogida de datos personales y se valoró el consumo de alcohol para identificar el tipo de consumo en los participantes a través del AUDIT; esto fue a manera de entrevista, respetando fielmente sus respuestas y sin emitir juicios al respecto. Cada AM, recibió retroalimentación sobre su forma de beber, a quienes se encontraron en consumo de riesgo (95 AM) se les invitó a participar en la intervención, aceptaron $50 \mathrm{AM}$.

Los participantes recibieron la intervención de enfermería que abordó: evaluación personalizada, retroalimentación, fijación de metas y monitoreo. La intervención en cuestión tuvo como fundamento la Terapia Racional Emotiva Conductual (TREC) ${ }^{(28)}$, dado que permite habilitar a las personas en el consumo responsable a través de cumplir sus propósitos-metas, enfocarse a permanecer vivo, obtener el mayor bienestar posible y evitar el malestar innecesario; aspectos importantes en la disminución del consumo de alcohol de riesgo en los adultos mayores. El diseño de la intervención estuvo a cargo de tres profesionales de enfermería y recibió retroalimentación por parte de un experto en diseño de intervenciones y uno en la TREC.

La metodología considerada para el diseño de la intervención fue la propuesta por Sidani y Braden ${ }^{(29)}$. La dosis de la intervención se determinó a partir de: la síntesis de evidencia, las recomendaciones para la aplicación de la TREC (28), las características propias de la población de AM y del estudio piloto de la intervención; obteniéndose nueve sesiones agrupadas en tres módulos, implementándose una sesión por semana con duración de 60 minutos 
efectivos. El propósito de la intervención fue habilitar a los participantes en el consumo responsable de alcohol. La implementación de la intervención fue por un facilitador con entremetimiento en la TREC y un equipo de logística.

En el primer módulo, se valoró el consumo de alcohol, se identificó el riesgo, se estableció el compromiso y objetivo por parte del AM hacía el consumo responsable de alcohol. En el segundo módulo, se trabajaron las destrezas y habilidades del AM para el consumo responsable, donde se identificaron los disparadores internos y externos del consumo de riesgo, se orientó sobre las estrategias de control de pensamientos, emociones y comportamientos, las acciones de autoayuda, como cuidarse a sí mismo y explorar su espiritualidad. En el tercer módulo, se abordó la motivación para prevenir recaídas, en el que se trabajó la inteligencia, la fuerza de voluntad, proyecciones hacia el futuro, momentos de inactividad-aburrimiento. En la tabla 1 se presentan las características generales de la intervención.

Tabla 1. Características de la intervención

\begin{tabular}{ll}
\hline Característica & Descripción \\
\hline Propósito & Habilitar a los participantes en el consumo responsable de alcohol \\
\hline Referente & Terapia Racional Emotiva Conductual (TREC) \\
\hline Contenido & $\begin{array}{l}\text { Módulo 1: riesgo de consumo de alcohol, compromisos y objetivos para el consumo } \\
\text { responsable de alcohol. } \\
\text { Módulo 2: destrezas y habilidades para el consumo responsable, disparadores del } \\
\text { consumo, estrategias de control, acciones de autoayuda } \\
\text { Módulo 3: motivación, inteligencia, fuerza de voluntad, proyecciones hacia el futuro }\end{array}$ \\
\hline Dosis y duración & Nueve sesiones, con duración de 60 minutos, se llevaron a cabo una por semana \\
\hline Modalidad de & Cara a cara individual \\
entrega & \\
\hline Escenario de entrega & Centro Gerontológico \\
\hline Requerimiento de & Facilitador con entremetimiento en la TREC, equipo de logística (3 integrantes) \\
equipo & Manual del facilitador y participantes.
\end{tabular}

Fuente: Elaboración propia 
Los datos se analizaron en el programa estadístico Statistical Package for the Social Sciences ${ }^{\circledR}$ (SPSS) versión

21, se realizó estadística descriptiva mediante frecuencias y porcentajes de las variables categóricas y respecto a las variables numéricas se obtuvieron medidas de tendencia central y dispersión. También, se realizó la prueba de bondad de ajuste de Kolmogorov-Smirnov con corrección de Lilliefors para contrastar la hipótesis de normalidad en la distribución de la variable, por lo que se determinó el uso de la prueba Wilcoxon para comparar el consumo pre y post intervención.

\section{Resultados}

En la tabla 2, se muestran las variables correspondientes a las características sociodemográficas de la población de estudio, donde se puede observar que $58 \%$ de adultos mayores corresponden al sexo femenino, de acuerdo con la escolaridad la mayor proporción (72\%) de participantes tenían estudios de primaria incompleta. Respecto a la edad de los participantes, osciló entre 60 y 71 años, con un promedio de 64.42 años $(D E= \pm 2.80)$.

Tabla 2. Características sociodemográficas de la población de estudio

\begin{tabular}{|c|c|c|c|}
\hline Variable & & $f$ & $\%$ \\
\hline \multicolumn{4}{|l|}{ Sexo } \\
\hline & Femenino & 29 & 58 \\
\hline & Masculino & 21 & 42 \\
\hline \multicolumn{4}{|c|}{ Escolaridad } \\
\hline & Primaria completa & 3 & 6 \\
\hline & Primaria incompleta & 36 & 72 \\
\hline & Secundaria incompleta & 8 & 16 \\
\hline & No fue a la escuela & 3 & 6 \\
\hline
\end{tabular}

En la tabla 3, se muestra la comparación de las mediciones pre y post intervención de la variable consumo de alcohol de riesgo en la que se observa significancia estadística $(Z=-6.160, p<.001)$ y la mediana fue menor en la medición post intervención. 
Tabla 3. Comparación del consumo de alcohol de riesgo pre y post intervención

Consumo de alcohol de riesgo

Pre intervención

Post intervención
12.62

7.16
Mediana

12.00

7.00
$D E$

1.67

$-6.160$

.933

Fuente: AUDIT

$n=50$

Nota: $D E=$ Desviación estándar, $Z=$ Prueba Wilcoxon

\section{Discusión}

En el presente estudio fue factible la aplicación empírica del concepto teórico de consumo de alcohol y la Terapia Racional Emotiva Conductual en adultos mayores con consumo de alcohol de riesgo.

Respecto, al efecto de la intervención se probó estadísticamente que fue significante $(Z=-6.16, p<.001)$, además la mediana fue menor en la medición post intervención (Mediana=7). Estos resultados tienen similitud con el estudio realizado en la Universidad de California que evaluó una intervención educativa para reducir el consumo de alcohol en adultos de 50 años y más. Los resultados reportaron diferencia significativa en los resultados del grupo de intervención vs control; bebedores con consumo de riesgo ( $66 \%$ vs $88 \%)$, consumo excesivo de alcohol ( $45 \%$ vs $68 \%{ }^{(19)}$. La similitud, de los resultados puede ser porque ambas intervenciones trabajaron en la retroalimentación sobre los riesgos específicos asociados con el consumo de alcohol de riesgo. Cuando el profesional de enfermería explica a la persona sobre los riesgos que conlleva su conducta actual a nivel personal y familiar se puede influir sobre la modificación de la conducta en la persona.

También, con los resultados de la investigación efectuada en Zurich, Suiza, que consistió en un programa para disminuir el consumo de alcohol en pacientes ambulatorios en edades de 50 a 65 años; se observó que $41.7 \%$ de los participantes del grupo control presentaron consumo de alcohol de riesgo en cambio en el grupo experimental fue 
28.6\% (OR=0.56, IC95\% 0.16-1.95, $p=.36)$ (20). Estos resultados guardaron semejanza con el estudio referido posiblemente porque en las dos intervenciones se incluyó monitoreo de los objetivos de consumo de alcohol. Al establecer, la meta de disminución de consumo de alcohol por la misma persona en el periodo que ella decida y tener el acompañamiento del profesional de enfermería puede garantizar el cumplimiento de la misma.

Además, con otro estudio realizado por la Universidad de California, que consistió en una intervención educativa para disminuir el riesgo de beber en adultos mayores de 60 años y más. En los resultados se observó asociación significativa en la disminución del consumo de riesgo ( $56 \%$ vs $67 \% ; p<.001)$, la cantidad de bebidas ingeridas $(-2.19 \text { bebidas por semana; } p<.001)^{(21)}$. La aproximación de los resultados con el estudio indicado es debido a que en ambas intervenciones se trabajó con diarios de consumo y orientación individual durante la sesión de la intervención. La utilización de un diario de consumo de alcohol permite a la persona conocer su avance entre el periodo de una sesión y otra, al recibir la orientación de enfermería la persona busca estrategias para acercase a la modificación de conducta.

Igualmente, con el estudio realizado al Sureste de California, que evaluó una intervención en adultos mayores de 55 y más años que decidieron cambiar su consumo de alcohol. En sus resultados, al terminar la intervención, se observó cambios en la cantidad de bebidas alcohólicas (14.9 vs. 12.1, $p<.05)$ y consumo de riesgo (2.8 vs. 2.1, p<. 001) (10). La similitud con el estudio mencionado es quizás porque en las dos intervenciones se trabajó en el mayor bienestar posible, esto al modificar la conducta de consumo de alcohol de riesgo a un consumo responsable. La persona logra reducir el consumo de alcohol al pensar que le beneficiará en su persona y contexto familiar.

\section{Conclusiones}

El estudio tuvo como objetivo evaluar el efecto de la intervención en la disminución del consumo de alcohol de riesgo en adultos mayores, con base a los hallazgos del estudio es posible concluir que la intervención mostró efecto en la disminución del consumo de riesgo de alcohol en los adultos mayores que participaron en la intervención. En este sentido la intervención presenta una alternativa factible para abordar la disminución del consumo de alcohol de riesgo en los adultos mayores. 
Con estos resultados el personal de salud del Centro Gerontológico tiene un punto de partida para dar seguimiento a los AM con consumo de alcohol de riesgo. De igual forma reforzar sus acciones a mantener su consumo responsable y así optimizar los recursos económicos de las instituciones en las que se aplique este tipo de intervenciones.

Es preciso señalar que el profesional de enfermería es un actor clave en los centros gerontológicos, porque además de realizar la detección del consumo de alcohol de riesgo interviene en su disminución. Por otra parte, dada la escasa producción científica sobre este tema, principalmente en México, el estudio contribuye en la generación de conocimiento sobre este fenómeno.

\section{Conflicto de intereses}

Los autores declaran la inexistencia de conflicto de intereses.

\section{Financiamiento}

El estudio no contó con financiamiento

\section{Referencias bibliográficas}

1. Bermúdez V, Torres Y, Apruzzese V, Martínez MS, Chavez M, Morillo J, Olivar L, Mejías J, Rojas M, Salazar J, Añez R, Rojas J. Alcohol driking patterns in the adult population from the Maracabio municipality, Zulia-Venezuela. Revista Latinoamericana de Hipertensión [Internet]. 2014 [consultado Febrero 2020]; 9(3): 21-28. Disponible en: https://www.redalyc.org/articulo.oa?id=170240766003

2. Guimaraes GL, Mendoza MÁ, López MÁ, García JÁ, Velasco-Ángeles LR, Beltrán MA, Valdez PE, Medina-Mora ME, Camacho R. Prevalencia y factores asociados al consumo de tabaco, alcohol y drogas en una muestra poblacional de adultos mayores del Distrito Federal. Salud Mental [Internet]. 2014 [consultado Febrero 2020]; 37(1): 15-25. Disponible en: http://www.scielo.org.mx/scielo.php?script=sci_arttext\&pid=S0185-33252014000100003\&lng=es.

3. Sacco P, Bucholz K, Harrigton D. Gender differences in stressful life events, social support, perceived stress, and alcohol use among older adults: Results from a national survey. Substance Use \& Misuse [Internet]. 2014 [consultado Febrero 2020]; (49): 456-465. Disponible en: http://dx.doi.org/10.3109/10826084.2013.846379

4. Immonen S, Valvanne J, Pitkälä KH. The prevalence of potential alcohol-drug interactions in older adults. Scandinavian journal of primary health care [Internet]. 2013 [consultado Febrero 2020]; 31(2): 73-78. Disponible en: http://dx.doi.org/10.3109/02813432.2013.788272

5. Mayhugh RE, Moussa MN, Simpson SL, Lyday RG, Burdette JH, Porrino LJ, Laurienti, PJ. Moderate-heavy alcohol consumption lifestyle in older adults is associated with altered central executive network community structure 
during cognitive task. PloS one [Internet]. 2016 [consultado Febrero 2020]; 11(8). Disponible en: http://dx.doi.org/10.1371/journal.pone.0160214

6. Aguila E, Guerrero EG, Vega WA. Sociodemographic characteristics associated with alcohol use among low-income Mexican older adults. Substance Abuse Treatment, Prevention, and Policy [Internet]. 2016 [consultado Febrero 2020]; (11):16. Disponible en: http://dx.doi.org/10.1186/s13011-016-0061-6

7. Marin JD. Consumo de alcohol en los adultos mayores: prevalencia y factores asociados. Boletín: Observatorio Chileno de Drogas [Internet]. 2015 [Consultado Febrero 2020]; (23): 1-5. Dispobinle en: http://www.senda.gob.cl/wp-content/uploads/2014/04/Boletin-N-\%C2\%A623-PDF2.pdf

8. Satre D, Bahorik A, Mackin R. Alcohol and drug use among older adults: associations with widowhood, relationship quality, and physical health, The Journals of Gerontology: Series B [Internet]. 2018 [consultado Febrero 2020]; 73(4): 633-635. Disponible en https://dx.doi.org/10.1093/geronb/gbx158

9. Natera G, Medina PS, Callegas F, Orford J, Salinas G, Tiburcio M. Costo-efectividad de una intervención breve de apoyo a mujeres indígenas de Hidalgo. México, que viven con consumidores excesivos de alcohol. Rev Panam Salud Publica [Internet]. 2016 [consultado Febrero 2020]; 39(2): 69-75. Disponible en: https://www.scielosp.org/article/rpsp/2016.v39n2/69-75/

10. Borok J, Galier P, Dinolfo M, Welgreen S, Hoffing M, Davis JW, Ramirez KD, Liao DH, Tang L, Karno M, Sacco P, Lin $J C$, Moore AA. Why do older unhealthy drinkers decide to make changes or not in their alcohol consumption? Data from the healthy living as you age study. Journal of the American Geriatrics Society [Internet]. 2013 [consultado Febrero 2020]; (61): 1296-1302. Disponible en http://dx.doi.org/10.1111/jgs.12394

11. Mazen D, Manzoor B, Lee T. Drug-alcohol interactions in olders U.S. adults. Journal The American Geriatrics Society [Internet]. 2015 [consultado Febrero 2020]; (63): 2324-2331. Disponible en http://dx.doi.org/10.1111/jgs.13787

12. Esmeraldas-Vélez ES, Falcones-Centeno MR, Vásquez-Zevallos MG, Solórzano-Vélez JA. El envejecimiento del adulto mayor y sus principales características. Revista Científica Mundo de la Investigación y el Conocimiento [Internet]. 2019 [consultado Septiembre 2020]; 3(1): 58-74. Disponible en: http://dx.doi.org/10.26820/recimundo/3.(1).enero.2019.58-74

13. Wolf IK, Du Y, Knopf H. Changes in prevalence of psychotropic drug use and alcohol consumption among the elderly in Germany: results of two National Health Interview and Examination Surveys 1997-99 and 2008-11. BMC psychiatry [Internet]. 2017 [consultado Febrero 2020]; 17(1): 90. Disponible en: http://dx.doi.org/10.1186/s12888017-1254-x

14. Gilson KM, Bryant C, Judd F. Exploring risky drinking and knowledge of safe driking guidelines in older adults. Substance Use \& Misuse [Internet]. 2014 [consultado Febrero 2020]; (49):1473-1479. Disponible en: http://dx.doi.org/10.3109/10826084.2014.912233

15. Instituto Nacional de Psiquiatría Ramón de la Fuente Muñiz, Instituto Nacional de Salud Pública, Comisión Nacional Contra las Adicciones, Secretaria de Salud, Villatoro, J.A. Resendiz, E. Mujica, A., Bretón, M., Cañas, V., Soto, I., Romero, M. y Mendoza, L. Encuesta Nacional de Consumo de drogas Alcohol y Tabaco 2016-2017: Reporte de Alcohol. México: INPRFM; 2017.

16. Pavón-León P, Gogeascoechea-Trejo MDC, Blázquez-Morales MLS, Sánchez-Solis A. Factores asociados con el consumo de alcohol y tabaco $\mathrm{n}$ adultos mayores. Revista Médica de la Universidad Veracruzana [Internet]. 2018 [consultado Febrero 2020]; 18(1): 7-16. Disponible en: https://www.medigraphic.com/pdfs/veracruzana/muv2018/muv181b.pdf 
17. Mendoza-Melendez MA, Borges GL, Gallegos-Cari A, García JA, Hernández-Llanes NF, Camacho-Solis R, MedinaMora ME. Asociación del consumo de sustancias psicoactivas con el cuidado y la salud del adulto mayor. Salud Mental [Internet]. 2015 [consultado Febrero 2020]; (38): 15-26. Disponible en: http://www.scielo.org.mx/scielo.php?script=sci_arttext\&pid=S0185-33252015000100003\&lng =es

18. Blow F, Barry K. Substance misuse and abuse in older adults: What do we need to know to help? Journal of the American Society on Aging [Internet]. 2014 [consultado Febrero 2020]; 38(3): 53-67. Disponible en: https://www.questia.com/library/journal/1P3-3539011491/substance-misuse-and-abuse-in-older-adults-what-do

19. Kuerbis AN, Yuan SE, Borok J, LeFevre P, Kim G, Lum D, Ramirez K, Liao D, Moore AA. Testing the initial efficacy of a mailed screening and brief feeback intervention to reduce at-risk drinking in middle-aged and older adults: The comorbidity alcohol risk evaluation (CARE) study. Journal of the American Geriatrics Society [Internet]. 2015 [consultado Febrero 2020]; 63(2): 321-326. Disponible en: http://dx.doi.org/10.1111/jgs.13237

20. Haug S, Lucht MJ, John U, Meyer C, Schaub MP. A pilot study on the feasibility and acceptability of a text messagebased aftercare treatment programme among alcohol outpatients. Alcohol Alcohol [Internet] 2015 [consultado Febrero 2020]; 50(2): 188-1894. Disponible en: http://dx.doi.org/10.1093/alcalc/agu107

21. Ettner SL, Su X, Duru K, Ang A, Tseng Ch, Tallen L, Barnes A, Mirkin M, Ransohoff K, Mooore AA. The effect of an educational intervention on alcohol consumption, at-risk driking and health care utilization in older adults: the Project SHARE study. Journal of studies on alcohol and drugs [Internet]. 2014 [consultado Febrero 2020]; 75(3): 447-457. Disponible en: http://dx.doi.org/10.15288/jsad.2014.75.447

22. Lefio LL, Villaroel SR, Rebodello C, Zamorano P, Rivas K. Intervenciones eficaces en consumo problemático de alcohol y otras drogas. Rev Panam Salud Publica [Internet]. 2013 [consultado Febrero 2020]; 34(4): 257-266.

23. Barry K, Blow F. Driking over the lifespan: Focus on older adults. Alcohol Res [Internet]. 2016 [consultado Febrero 2020]; 38(1): 115-120. Disponible en: https://www.ncbi.nlm.nih.gov/pmc/articles/PMC4872606/

24. Crome I, Li T, Rao R, Wu L. Alcohol limits in older people. Addiction [Internet]. 2012 [consultado Febrero 2020]; (107): 1541-1543. Disponible en: http://dx.doi.org/10.1111/j.1360-0443.2012.03854.x

25. Cohen J. Statistical power analysis for the behavioral sciences (2nd ed.). Hillsdale, NJ: Lawrence Erlbaum Associates; 1988

26. De la Fuente J, Kershenobich D. El alcoholismo como problema médico. Revista Facultad de Medicina UNAM, 1992; 35(2): 47-51

27. Aguilar-Navarro SG, Reyes-Guerrero J, Borgues G. Alcohol, tabaco y deterioro cognoscitivo en adultos mexicanos mayores de 65 años. Salud pública Méx [Internet]. 2007 [consultado Septiembre 2020]; 49(4): 467-474. Disponible en: http://www.scielo.org.mx/scielo.php?script=sci_arttext\&pid=S0036-36342007001000005\&lng=es.

28. Ellis A. Rational Emotive Behavior Therapy and the Mindfulness. Based Stress Reduction Training of Jon KabatZinn. Journal of Rational Emotive and Cognitive Behavior Therapy [Internet]. 2006 [consultado Febrero 2020]; 24 (1), 63-78. Disponible en: http://dx.doi.org/10.1007/s10942-006-0024-3

29. Sidani S, Braden CJ. Desing, evaluation and translation of nursing interventions (2nd ed.). USA: Wiley-Blackwell; 2011

Cómo citar este artículo: Álvarez-Aguirre A, Casique-Casique L, López-Nolasco B, Tolentino-Ferrel MR. Efecto de una intervención de enfermería para la disminución del consumo de alcohol de riesgo en adultos mayores. SANUS [Internet]. 2021 [citado el $\underline{\mathrm{dd}} \underline{\mathrm{mm}}$ aa];6:e195. Disponible en: DOI/Link 